\title{
Current Status of Staged Reconstruction for Hypoplastic Left Heart Syndrome
}

\author{
E.L. Bove \\ Pediatric Cardiovascular Surgery, The University of Michigan Health System, Ann Arbor, MI 48109, USA
}

\begin{abstract}
Once considered a uniformly fatal condition, the outlook for newborns with hypoplastic left heart syndrome has been dramatically altered with staged reconstructive procedures. Refinements in operative technique and perioperative management have been largely responsible for this improved outlook. At the University of Michigan, 253 patients underwent the Norwood operation for classic hypoplastic left heart syndrome between January 1990 and November 1997. Hospital survival was $76 \%$. Among patients considered at standard risk, survival was significantly higher $(86 \%)$ than that for those patients with important risk factors $(42 \%, p=0.0001)$. Adverse survival was most strongly associated with significant associated noncardiac congenital conditions $(p$ $=0.008$ ) and severe preoperative obstruction to pulmonary venous return $(p=0.03)$. Survival following second-stage reconstruction with a hemi-Fontan or bidirectional Glenn procedure was $97 \%$. The Fontan procedure has been completed in 94 of these patients with a hospital survival rate of $88 \%$. Survival after the Fontan procedure improved significantly when the second stage of the reconstruction was completed with a hemi-Fontan procedure compared to a bidirectional Glenn ( $98 \%$ vs $81 \%, p$ $=0.05)$. Among the patients considered at standard risk, actuarial survival was $70 \%$ at 5 years. The largest decrease in survival occurred in the first month of life and late deaths affected primarily those patients in the highrisk group. Neurodevelopmental outcome studies demonstrated normal verbal and performance scores in the majority of patients. Staged reconstruction has significantly improved the intermediate-term outlook for patients with hypoplastic left heart syndrome. Factors addressing improvements in early first-stage survival would be expected to add significantly to an overall improved late outcome.
\end{abstract}

Key words: Hypoplastic left heart syndrome

Refinements in operative technique and perioperative care have been largely responsible for an improved out- look for newborns with hypoplastic left heart syndrome (HLHS). Staged reconstruction for patients with HLHS is based on the knowledge that an effective circulation is possible in the absence of a pulmonary ventricle. The principle that pulmonary blood flow could be maintained when certain well-defined hemodynamic criteria were met has led to the application of the Fontan procedure to virtually all forms of single ventricle lesions [7]. The first stage in the reconstructive process, the Norwood procedure, must provide (1) unobstructed systemic and coronary blood flow from the right ventricle, (2) unobstructed pulmonary venous return across the atrial septum, and (3) sufficient pulmonary blood flow without significant volume overload [14, 20,21]. This leaves the pulmonary and systemic circulations in parallel and the right ventricle to perform the increased volume work of both circulations. The second-stage procedure, the hemi-Fontan or bidirectional Glenn operation, results in removal of the ventricular volume overload imposed by the systemic shunt and the connection of the superior vena cava to the pulmonary arteries [5, 22]. Augmenting the central pulmonary arteries, preventing conduction disturbances, and constructing a potential connection for the inferior vena cava to the pulmonary arteries are considered important components of this procedure $[1,4]$. The hemi-Fontan procedure, currently preferred to the bidirectional Glenn at this institution, also allows for the correction of additional risk factors, including pulmonary artery distortion, and maintains a stable circulation free of right ventricular volume overload and pulmonary hypertension. During the last stage of reconstruction in the Fontan procedure, inferior vena caval return is channeled to the pulmonary arteries to complete the separation of the pulmonary and systemic circulations. Although the current techniques have resulted in substantial improvements in the quantity and quality of survival, efforts to refine each stage of the process continue to evolve with increasing follow-up and evaluation $[4,12,18]$.

Many institutions, initially discouraged by the poor results of palliative reconstructive surgery for hypoplastic left heart syndrome, abandoned this approach in favor of transplantation [11, 23]. However, the lack of avail- 
able donor hearts, late complications of cardiac transplantation, and the improvements noted with staged reconstruction have again encouraged an increasing number of centers in North America and elsewhere to reevaluate this form of therapy. At the University of Michigan, we have continued to pursue a policy of offering staged reconstructive surgery for patients with HLHS. The evolution of our approach and the lessons learned in this endeavor are presented.

\section{Methods}

\section{Initial Management and the Norwood Procedure}

The majority of newborns with HLHS are well palliated with an infusion of prostaglandin and reduction of the inspired oxygen concentration to avoid high systemic arterial saturations, but this condition cannot be sustained indefinitely and a progressive deterioration in pulmonary function characterized by increasing pulmonary edema will become evident with time. The presence of significant obstruction to pulmonary venous return, most commonly resulting from a restrictive atrial septal defect, will hasten this outcome. In general, when systemic arterial oxygen saturation is in excess of $80 \%$, peripheral perfusion is well maintained, and the chest radiograph shows little or no pulmonary edema, most patients will remain stable and may undergo operation electively within a few days. However, more significant degrees of obstruction to pulmonary venous return will result in severe and rapidly progressive pulmonary edema with systemic arterial oxygen saturations falling below $75-80 \%$. Urgent intervention is often required before the pulmonary status deteriorates further. Percutaneous balloon septostomy, often requiring a bioptome to traverse the thickened atrial septum, has been effective in our laboratory. Operation can then be postponed for a few days to allow the pulmonary edema time to resolve. It has been our experience that optimal results following the Norwood procedure are obtained when pulmonary blood flow is restricted with the use of a small shunt $[13,16]$. However, satisfactory postoperative oxygen saturations are increasingly difficult to maintain when a small shunt is used in a patient with pulmonary edema. A larger shunt will be required that will often result in pulmonary overcirculation after only a few hours following cardiopulmonary bypass as the pulmonary edema and elevated pulmonary vascular resistance resolves. A rapid deterioration in systemic perfusion associated with increasing pulmonary blood flow may result in hypotension, metabolic acidosis, and death. A significant diastolic runoff of blood into the pulmonary circulation will also occur with a large shunt, further adding to low cardiac output from impaired coronary blood flow due to low diastolic blood pressure [6].

Surgical Technique. Although the method of surgical repair has remained essentially constant over the past few years, small modifications have greatly improved the quality of the outcome. These modifications include (1) accurate orientation of the anastomosis between the ascending aorta and the proximal main pulmonary trunk, (2) augmentation of the descending aorta well beyond the ductus insertion, (3) improved tailoring of the patch of pulmonary allograft tissue used for the arch augmentation, and (4) the use of smaller shunts. Cardiopulmonary bypass is established by cannulation of the proximal main pulmonary artery just distal to the pulmonary valve and the right atrial appendage. The branch right and left pulmonary arteries are occluded and systemic cooling is begun in preparation for circulatory arrest. The ascending, transverse, and proximal descending segments of the aorta are mobilized and tourniquets are placed around each head artery for subsequent occlusion. A cryopreserved pulmonary allograft is trimmed

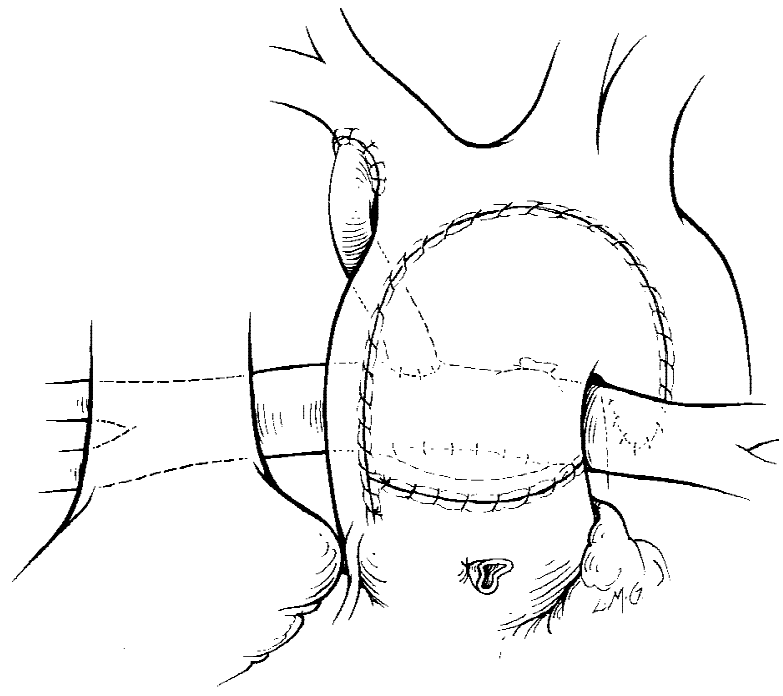

Fig. 1. Reconstruction following the Norwood procedure demonstrates the shunt, which is positioned between the distal innominate or proximal subclavian artery and the central pulmonary arteries. Appropriate tailoring of the allograft patch is essential to avoid excessive dilatation of the ascending aorta and compression of the left pulmonary artery. Reprinted with permission from Bove and Mosca [4].

to fashion a patch that will serve to augment the aorta and allow anastomosis to the proximal main pulmonary trunk. Following the induction of circulatory arrest, the atrial septum is excised through the atrial appendage purse string or a short right atriotomy. The main pulmonary trunk is then divided at the bifurcation and the distal end closed with a synthetic patch of GoreTex. The ductal tissue is then excised completely and the resultant opening is extended approximately $10 \mathrm{~mm}$ further distally into the descending aorta. This incision is then extended proximally under the transverse arch and down the diminutive ascending aorta until the level of the previously divided main pulmonary trunk is reached. The ascending aorta is connected to the main pulmonary artery with interrupted sutures in the proximal corner near the coronary arteries and the remainder of the aorta is augmented with the previously cut pulmonary allograft patch. A 3.5$\mathrm{mm}$ Gore-Tex conduit is anastomosed from the subclavian or distal innominate artery to the central pulmonary artery during the rewarming (Fig. 1). A 4-mm conduit is occasionally used for patients weighing over approximately $4 \mathrm{~kg}$, those with significantly increased extravascular lung water, or those who are beyond the first month of life. The distal end of the shunt is placed close to the divided end of the ductus rather than onto the right pulmonary artery itself.

\section{The Second Stage: Hemi-Fontan Procedure}

At the University of Michigan, the hemi-Fontan procedure is preferred to the bidirectional Glenn because of the capacity to more easily augment the central pulmonary arteries and to provide a large eventual connection for the inferior vena cava at the Fontan procedure. Although more complex, the hemi-Fontan procedure significantly simplifies the eventual Fontan, at which time optimal hemodynamics and pulmonary function are most essential to outcome. The hemi-Fontan procedure is generally performed between 4 and 6 months of age. Cardiac catheterization is routinely performed to assess (1) right ventricular function, 


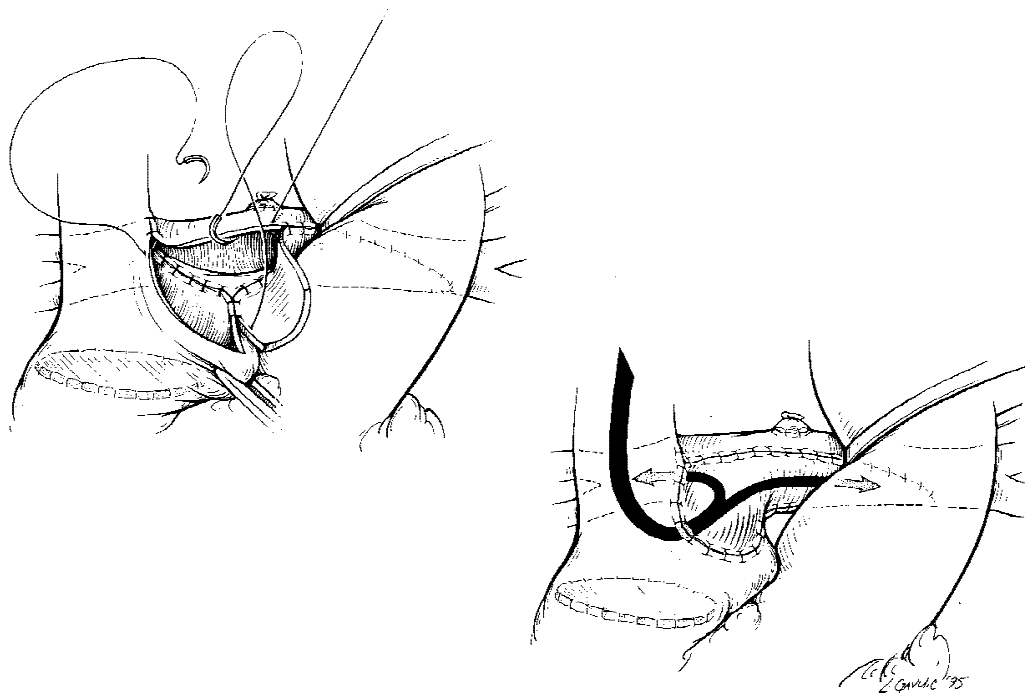

Fig. 2. The steps for the second-stage reconstruction or the hemi-Fontan procedure. The central pulmonary arteries are opened anteriorly and an incision is made along the base of the right atrial appendage (left). A patch is fashioned from a cryopreserved pulmonary allograft and used to augment the central pulmonary arteries (right). An additional patch is placed within the right atrium to exclude the superior vena cava and the pulmonary arteries from the heart. Although not illustrated in this figure, the atrial incision does not cross the cavoatrial junction to avoid injuring the artery to the sinoatrial node. Reprinted with permission from Bove and Mosca [4].
(2) tricuspid regurgitation, (3) residual arch or pulmonary venous (atrial septal) obstruction, (4) pulmonary artery anatomy, and (5) pulmonary vascular resistance. The number of patients with contraindications to proceeding with the second-stage procedure has been surprisingly few. Absolute contraindications have included isolated poor right ventricular function and elevated pulmonary vascular resistance. However, when decreased right ventricular function exists in association with another correctable problem, typically residual arch obstruction, excessive volume overload from a large shunt, or tricuspid valve regurgitation, we have proceeded with the hemi-Fontan with the expectation that ventricular function will improve. The belief that tricuspid valve regurgitation was secondary to volume overload and that it would improve after the second-stage procedure has not proven to be the case, and we now perform tricuspid valvuloplasty at the hemi-Fontan procedure when regurgitation is more than mild [24]. Although placement of the shunt on the central pulmonary artery near the ductal insertion has resulted in more symmetric growth of the pulmonary arteries following the Norwood procedure, it has been common to find mild pulmonary artery narrowing at the origin of the left pulmonary artery. The use of pulmonary allograft patches of smaller widths at the time of the Norwood procedure has also improved the arch reconstruction and reduced the incidence of compression of the left pulmonary artery.

Surgical Technique. Cannulation for cardiopulmonary bypass is accomplished through the distal ascending aorta, placing the arterial cannula through allograft patch tissue and the venous cannula through the mid-right atrial wall. The shunt is divided and the pulmonary arteries are mobilized widely. A patch of pulmonary allograft material is fashioned for augmentation of the pulmonary arteries. The superior vena cava is mobilized and the azygous vein is ligated. The central pulmonary arteries are opened beginning beneath the superior vena cava and extending nearly to the left upper lobe branch (Fig. 2). An incision is made in the base of the right atrial appendage but is stopped before its junction with the superior vena cava. This incision has been modified to avoid injuring the artery to the sinoatrial node. The pulmonary artery is sutured to the outside wall of the superior vena cava until the right atrial incision is reached, transferring the suture line to the atriotomy itself. The pulmonary arteries are then enlarged with the allograft patch. A separate patch is placed within the right atrium, at the level of the limbus of the septum secundum, which will isolate superior vena caval return into the pulmonary arteries and provide an unobstructed pathway for the connection of inferior vena caval return at the time of the Fontan procedure. Prior to the insertion of this patch, the atrial septal defect is inspected and enlarged if necessary. This is best accomplished by cutting back the coronary sinus into the left atrium. If indicated, tricuspid valve repair is also performed at this time.

\section{The Third Stage: The Fontan Procedure}

The majority of patients following the second-stage reconstruction have maintained satisfactory palliation for a considerable period of time [5]. However, some will experience a progressive decline in systemic arterial oxygen saturation with the development of venovenous collaterals to the inferior vena caval circulation. Additionally, increasing systemic hypoxemia will be noted in some patients as a result of increasing growth and activity leading to an increased return of desaturated lower extremity blood with exercise. Even those patients with azygous continuation of an interrupted inferior vena cava have experienced a progressive fall in oxygen saturation as venous collaterals form to the hepatic venous circulation. The development of arteriovenous malformations may constitute another important etiology for progressive hypoxemia in this group [26]. Additionally, chronic hypoxemia will stimulate growth and development of systemic arterial collaterals into the pulmonary circulation resulting in increased volume overload that may complicate the postoperative course after the Fontan procedure. For these reasons, the Fontan procedure has generally been performed at approximately 18 months to 2 years of age. Preoperative evaluation, including Doppler/echocardiography and cardiac catheterization, is essential to assess ventricular function, atrioventricular valve regurgitation, pulmonary artery size, pressure and resistance, and the presence of systemic to pulmonary artery collateral vessels. Major collaterals should be occluded by coil embolization preoperatively. The size of the left pulmonary artery is of potential concern following staged reconstruction for HLHS (Fig. 3). A dilated neoaorta following the Norwood procedure may cause compression and subsequent hypoplasia of the left pulmonary artery. In addition, the low pressure and reduced flow situation imposed by the hemi-Fontan procedure may result in a left pulmonary artery of insufficient size for the Fontan. Insertion of an endoluminal stent at the time of operation has proved to be a useful adjunct in a few of our patients with otherwise refractory hypoplasia of the left pulmonary artery. We prefer to utilize stents 


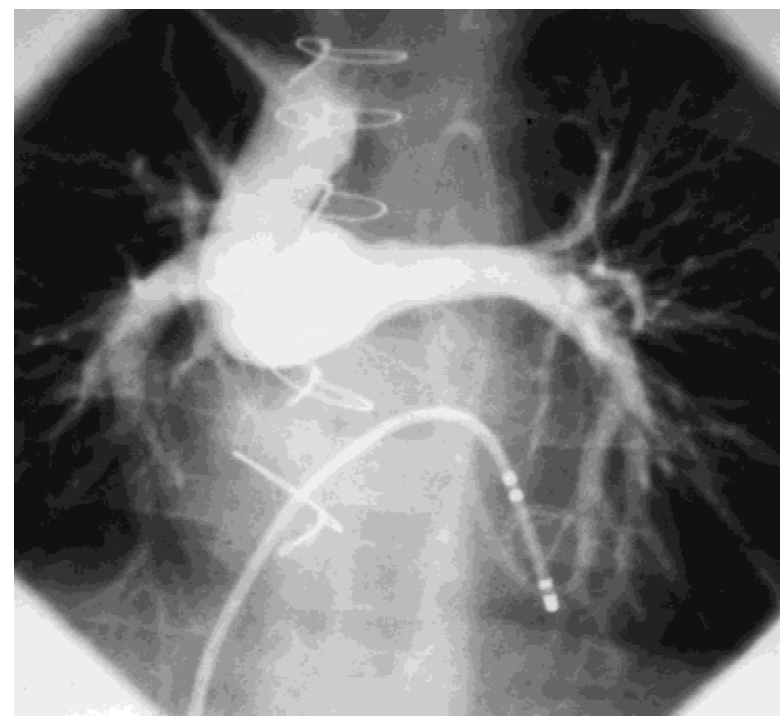

Fig. 3. Angiogram performed with contrast injected into the superior vena cava following the hemi-Fontan procedure. Unobstructed pulmonary arteries are visualized and no contrast enters the right atrium. Reprinted with permission from Bove and Mosca [4].

sparingly and, when necessary, to place them at the final procedure when a larger size stent can be safely inserted.

Surgical Technique. The heart is exposed through a midline sternotomy, dissecting adhesions sparingly to avoid injury to the phrenic nerves. We have not used pericardial substitutes to facilitate reoperation and have found little difficulty with sternal reentry. The majority of the Fontan procedures were initially performed on continuous cardiopulmonary bypass with bicaval venous return. However, currently single venous return is accomplished through the right atrium and the procedure is done during a brief period of circulatory arrest. The substantially reduced operative dissection required for this approach has been possible because of the preliminary connections made at the hemiFontan operation [19]. The entire operative procedure and, in particular, the cardiopulmonary bypass and aortic cross-clamp times have been reduced considerably (Table 1). The right atrium is opened and the previously placed patch between the superior vena cava-pulmonary artery connection and the right atrium is removed (Fig. 4). A Gore-Tex patch is then fashioned to channel inferior vena caval return to the pulmonary arteries (Figs. 5 and 6). An extracardiac conduit has not been used in our institution except in circumstances in which a lateral tunnel cannot be constructed (e.g., anomalous pulmonary venous connection and certain heterotaxy syndromes). A fenestration of $4 \mathrm{~mm}$ in diameter has been performed in our institution for all high-risk patients undergoing the Fontan operation.

\section{Results}

A retrospective study was recently performed to evaluate the outcome of all patients with the diagnosis of HLHS who presented to C.S. Mott Children's Hospital, University of Michigan Health System, and underwent firststage reconstructive surgery with a Norwood procedure between January 1990 and August 1995 [3, 15]. In order
Table 1. Cardiopulmonary bypass and cross-clamp times for the Fontan procedure.

\begin{tabular}{lll}
\hline $\begin{array}{l}\text { Type of } \\
\text { second stage }\end{array}$ & $\begin{array}{l}\text { Cardiopulmonary } \\
\text { bypass time } \\
\text { (minutes) }\end{array}$ & $\begin{array}{l}\text { Cross-clamp } \\
\text { time } \\
\text { (minutes) }\end{array}$ \\
\hline $\begin{array}{l}\text { Bidirectional Glenn } \\
\text { Hemi-Fontan }\end{array}$ & $\begin{array}{r}111 \pm 32 \\
56 \pm 10\end{array}$ & $59 \pm 19$ \\
\hline
\end{tabular}

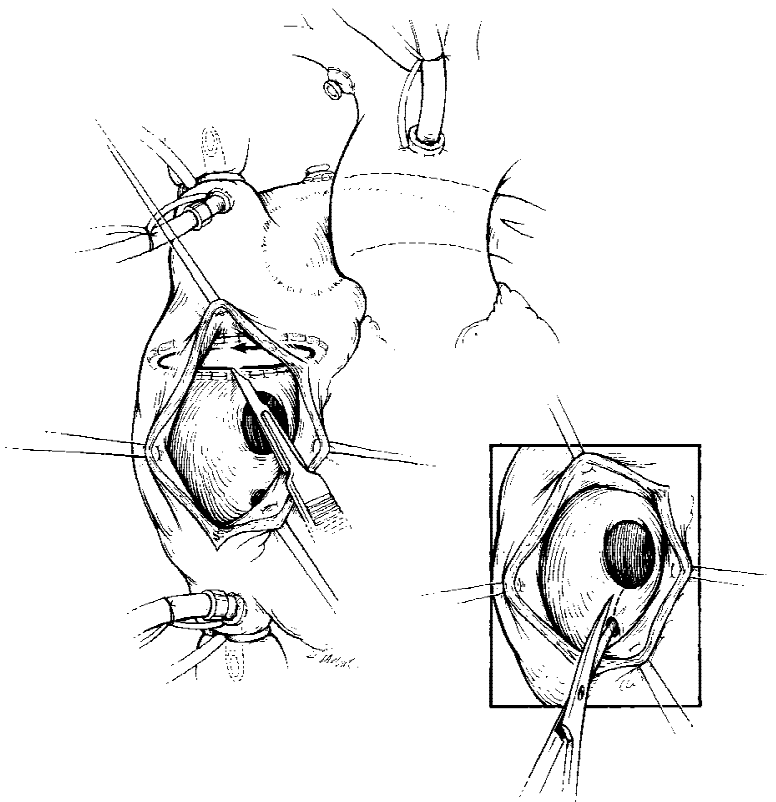

Fig. 4. The Fontan procedure is performed by first removing the previously inserted patch beneath the superior vena caval-pulmonary artery connection. The coronary sinus is cut back to enlarge the atrial septal defect if necessary (insert). Reprinted with permission from Bove and Mosca [4].

to obtain an accurate assessment of the results for those patients with a single, isolated malformation, only patients with classic HLHS were included for analysis. For the purposes of that review, classic HLHS was defined as a right ventricular-dependent circulation in association with atresia or severe hypoplasia of the aortic valve. Therefore, patients with hypoplastic left ventricles but with an otherwise adequate aortic outflow tract (e.g., transposition of the great arteries with mitral atresia) were excluded, as were patients with aortic atresia or hypoplasia but with an adequate left ventricle (e.g., double-inlet left ventricle with aortic atresia). This was true even though a Norwood procedure was performed. Additionally, those patients with double-outlet right ventricle, atrioventricular septal defect, and atrioventricular discordance were excluded from analysis, except in the rare instance in which both left ventricular and aortic 

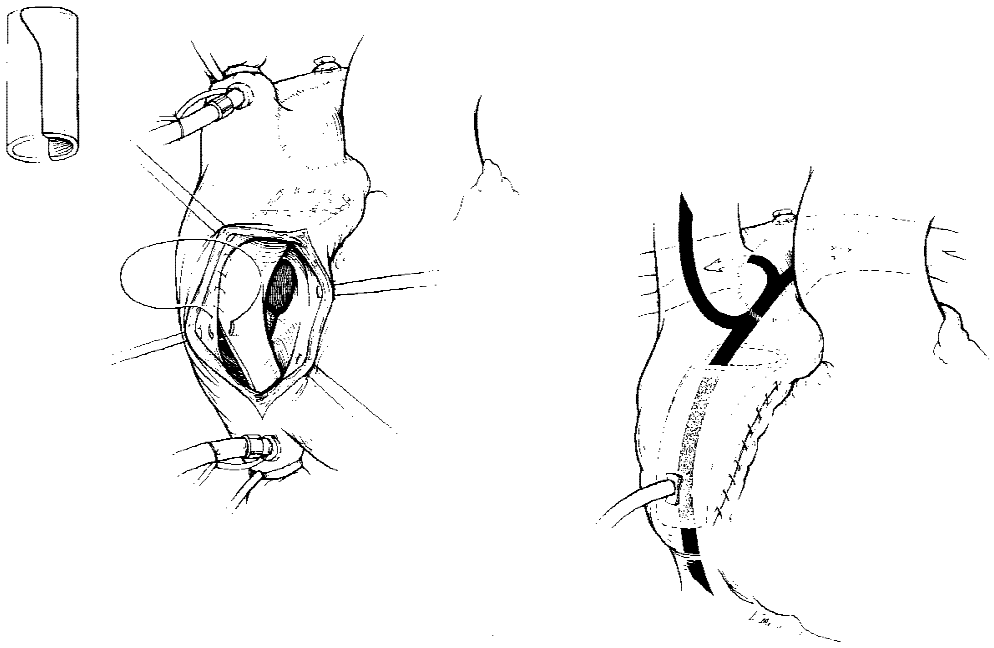

Fig. 5. A polytetrafluoroethylene patch is used to channel inferior vena caval return to the previously constructed anastomosis with the pulmonary arteries. Alternatively, the patch may be cut from a conduit opened along one side (left). The external position of the snare surrounding the fenestration is shown (right). Reprinted with permission from Bove and Mosca [4].

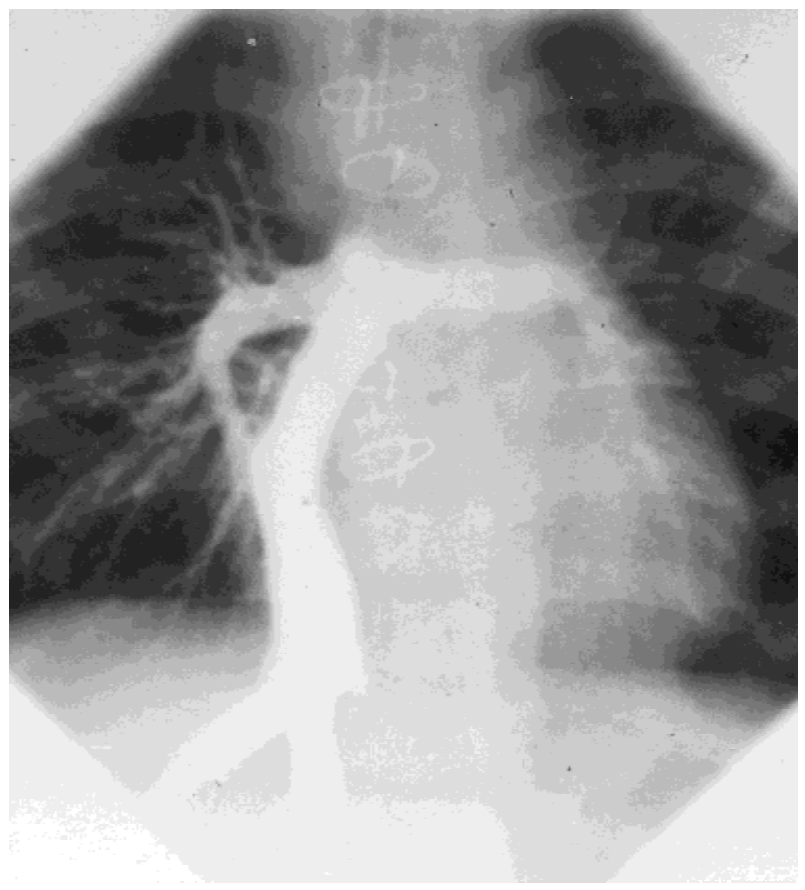

Fig. 6. Angiogram performed after the Fontan procedure demonstrating the completed cavopulmonary connection. A streamlined pathway from the inferior vena cava is shown. Reprinted with permission from Bove and Mosca [4].

atresia or hypoplasia existed together. Patients in the study were divided into "standard-risk" and "highrisk" groups according to certain predefined criteria established from prior analyses performed at this institution $[13,15]$.

\section{Definition of Risk Groups}

In order to define the high-risk population, patients presenting with coexisting conditions known to affect out- come were considered as a separate group. Considered among the high-risk group were patients undergoing the Norwood procedure beyond the first month of life and those presenting with severe obstruction to pulmonary venous return. The latter was defined as the occurrence of severe hypoxemia in conjunction with radiographic evidence of pulmonary edema and an intact or nearly intact atrial septum confirmed by Doppler/echocardiography and by direct surgical or pathologic inspection. Finally, patients with significant noncardiac congenital conditions that were judged to affect or potentially affect prognosis were also excluded from the standard-risk group. Such conditions included prematurity ( $<35$ weeks' gestational age), low birth weight $(<2.5$ $\mathrm{kg}$ ), chromosomal anomalies, and major noncardiac anomalies such as diaphragmatic hernia. The remaining patients were considered at standard risk, but this group was anatomically diverse and, therefore, patients with coexisting anomalies of the ventricular or atrioventricular septum, visceroatrial situs defects, or atrioventricular discordance were analyzed separately. Patients were not excluded for systemic or pulmonary venous or arterial anomalies. Because prior reports from this institution failed to identify the specific morphologic subgroup (i.e., aortic atresia and mitral atresia, aortic atresia and mitral hypoplasia, aortic hypoplasia and mitral atresia, and aortic hypoplasia and mitral hypoplasia) as a risk factor, no further anatomic subdivision of the standard-risk group was undertaken for the current analysis. Patients were not stratified by preoperative condition. Therefore, the standard-risk group was composed of patients with typical HLHS, including those with minor anatomic variants, who underwent primary Norwood operation within the first month of life and who did not have severe obstruction to pulmonary venous return or significant noncardiac conditions. All other patients were analyzed in the high-risk group. 


\section{Clinical Data}

Between January 1990 and November 1997, 253 patients with classic HLHS underwent a Norwood procedure at the University of Michigan, exclusive of those patients undergoing Norwood procedures for other single ventricle lesions with systemic outflow tract obstruction. A detailed analysis of the first 158 of these patients operated upon between January 1990 and August 1995 was previously reported [3]. Between September 1995 and November 1997, an additional 95 patients with the classic form of HLHS, both standard and high risk, underwent the Norwood procedure at our institution, bringing the total to 253 patients. Hospital survival for this latter group has been identical to that of patients in the previous study. Because long-term analysis is not yet available for these recent patients and their outcomes appear to be no different from those of the earlier group, the data reported here are from our prior report.

\section{Survival}

Of the 158 patients undergoing the Norwood procedure, there were 120 hospital survivors [76\%; 70\% confidence limit (CL70), 72-79\%). Of note, hospital survival for the most recent 95 patients not included in the earlier review, both standard and high risk, was $75 \%$. Hospital survival was significantly better among the 127 standard risk patients (86\%; CL70, 82-89\%) when compared to those in the high-risk group (42\%; CL70, 33-51\%; $p=0.0001$ ). Patients were excluded from the standard-risk group because of associated noncardiac congenital conditions in 17 , severe pulmonary venous obstruction in 8 , and age beyond 1 month at initial surgical intervention in 6 . Further analysis of the high-risk group revealed that hospital survival following the Norwood procedure was significantly worse among those patients with significant noncardiac congenital conditions $(p=0.008)$ and in those with severe obstruction to pulmonary venous return $(p=$ $0.03)$.

There were 106 hemi-Fontan procedures performed with 103 hospital survivors (97\%; CL70, 95-98\%). There was 1 late death. Three of the survivors are not considered to be candidates for the Fontan procedure because of neurologic dysfunction (pre-Norwood), pulmonary artery distortion unresponsive to surgical repair and endoluminal stenting, and right ventricular dysfunction following an influenza-like illness, respectively. The latter patient has undergone successful cardiac transplantation.

In a report from this institution, the outcome of the 94 patients in the group following the Fontan procedure was reviewed [17]. Overall survival was $88 \%$ [95\% confidence limit (CL95); 81-94\%]. Survival was significantly better among the 42 patients who underwent a preliminary hemi-Fontan operation (98\%; CL95, 88$100 \%)$ when compared to those undergoing a bidirectional Glenn procedure (81\%; CL95, 76-86\%; $p<0.05)$.

The largest decrease in survival occurred in the first month of life. Losses among survivors of the Norwood procedure prior to or related to the hemi-Fontan procedure affected primarily the high-risk group and deaths occurring during the second and third year of life were primarily due to hospital death following the Fontan operation. The latter was due primarily to unrecognized stenosis or hypoplasia of the left pulmonary artery and occurred early in our experience before routine use of the hemi-Fontan procedure. Survival at 1 month, 1 year, and 5 years for the 127 patients considered at standard risk and with typical anatomy was $85 \pm 4 \%, 80 \pm 5 \%$, and 69 $\pm 8 \%$, whereas survival for those with anatomic variants was $86 \pm 11 \%, 71 \pm 17 \%$, and $71 \pm 17 \%$, respectively. Survival for high-risk patients was $61 \pm 14 \%, 20 \pm 40 \%$, and $20 \pm 40 \%$ at 1 month, 1 year, and 2 years, respectively.

\section{Morbidity}

Significant or potentially significant harmful conditions were noted in 25 of the 120 hospital survivors (21\%; CL70, 17-25\%). Morbidity related to the cardiovascular system was noted in 12 patients (10\%; CL70, 8-13\%). Harmful conditions included dysrhythmias in 5 patients (3 with permanent pacemakers, 1 with supraventricular tachycardia requiring amiodarone, and 1 with sick sinus syndrome), left pulmonary artery occlusion in 4 patients (patency restored in 3), chronic pleural effusions in 2, pulmonary artery hypertension requiring oxygen therapy in 1 patient in whom both the Norwood and hemi-Fontan procedures were performed after awaiting a transplant for 7 months at another institution, and dilated cardiomyopathy requiring transplantation at age 27 months in 1 patient. One patient had both dysrhythmias and pulmonary artery occlusion. Mechanical ventilation was required in 4 patients, with only 1 late survivor. Hemidiaphragm paralysis was noted in 7 patients, 6 of whom underwent plication.

\section{Neurodevelopmental Outcome}

Late neurodevelopmental outcome was assessed in 49 patients following the Fontan procedure in a recent study from our institution [10]. Wechsler Preschool and Primary Scales of Intelligence-Revised were used for patients between 34 and 72 months of age and Wechsler Intelligence Scales for Children for those between 73 and 96 months. Mean verbal, performance, and overall test scores were normal for the 25 patients in this study with HLHS, although scorers were slightly lower than those 
of patients with other single ventricle lesions. For both groups, verbal and communication skills were significantly and consistently better than motor and performance skills.

\section{Discussion}

The term hypoplastic left heart syndrome, first used by Hauck and Nadas in 1958, has remained a clinically useful description of a constellation of cardiac malformations all characterized by underdevelopment or the absence of the left ventricle. The ability to make a precise diagnosis in the newborn and the introduction of prostaglandin $E_{1}$ to maintain patency of the ductus arteriosus to support the systemic circulation allowed the development of palliative surgical approaches. The majority of babies with HLHS are otherwise normal newborns. Only $5 \%$ are premature or small for gestational age. Noncardiac malformations coexist in $12 \%$ and are major in only $2 \%$ or $3 \%$. Without treatment, $95 \%$ die within the first month of life. The postnatal physiology is characterized by an obligatory left to right shunt at atrial level and the need for the right ventricle to supply both the parallel systemic and pulmonary circulations [10]. The coronary and brachiocephalic vessels are perfused in a retrograde fashion. Alteration in hemodynamic status is determined by three major factors: (1) the expected fall in pulmonary vascular resistance, (2) constriction of the ductus arteriosus, (3) and the adequacy of the atrial communication. Stabilization of the newborn with HLHS is, consequently, dependent on the status and/or ability to favorably influence these factors.

There is no doubt that staged reconstruction now offers survival for patients with HLHS that equals that currently reported for many other forms of complex congenital cardiac malformations. In our series, the actuarial survival rate, including hospital death for each operative procedure, for those patients considered at standard risk is approximately $70 \%$ at 5 years. These data compare favorably to those for patients with other forms of single ventricle, pulmonary atresia with intact ventricular septum, and even for some complex forms of tetralogy of Fallot. High-risk patients, on the other hand, did not fare as well. For these patients, it may be reasonable to conclude that cardiac transplantation should be offered instead of staged reconstruction. However, the often grave and unstable condition of most of these patients would clearly preclude them from transplantation as a viable option. In an effort to improve the results among this group of patients, those presenting with severe obstruction to pulmonary venous return now undergo preliminary atrial septectomy, either percutaneous or operative, and the Norwood procedure is postponed until evidence of diminished pulmonary vascular resistance and lung fields free of edema are seen. Patients below $2.5 \mathrm{~kg}$ in weight often receive a standard Blalock-Taussig shunt which appears to limit pulmonary blood flow to a more appropriate degree and is better tolerated in very small birth weight babies. Because the greatest risk for death occurs during the first month of life, efforts to reduce perioperative Norwood mortality would be expected to result in improved late survival. Survival following the hemi-Fontan procedure has been excellent and late sudden death unrelated to subsequent operations has been rare. Additionally, mortality associated with the Fontan procedure occurred almost entirely with the earliest group of patients coming to the third stage. This risk has been substantially reduced in recent patients.

It is often difficult to be certain that postoperative neurologic and/or cardiovascular conditions are a result of any given operation or intervention because many of these patients were gravely ill upon presentation and prior to any procedures $[8,9,25]$. Nonetheless, previous attempts to correlate preoperative condition to outcome failed to reveal an association and many patients who were moribund prior to their Norwood procedure achieved excellent late results [13]. The effects of the operative procedures, including the use of deep hypothermia and circulatory arrest, are unclear but are currently undergoing detailed evaluation. In a recent report from our institution, Goldberg and colleagues [10] found that neurodevelopmental outcome was normal in the majority of patients evaluated after the Fontan procedure. Although the group of patients with HLHS fared slightly less well than other patients with single ventricle lesions, most remained within the normal range. Morbidity related to the cardiovascular and respiratory systems will undoubtedly always be a part of these complex surgical interventions, but the modifications noted earlier have significantly reduced their impact.

\section{Conclusions}

Staged reconstruction for neonates born with HLHS offers a viable form of treatment with a good intermediateterm outcome. Survival has substantially improved and morbidity surrounding various interventions has been significantly reduced. Although it appears that most survivors grow and develop normally, it is essential to continually evaluate these patients to assess late quality of life. Current efforts aimed at reducing mortality following the Norwood operation by providing a more stable circulation and at eliminating the risk factors for the Fontan procedure are expected to result in continued improvements in late survival.

\section{References}

1. Alboliras ET, Chin AJ, Barber G (1989) Pulmonary artery configuration after palliative operations for hypoplastic left heart syndrome. J Thorac Cardiovasc Surg 97:878-885

2. Barnea O, Austin EH, Richman B, Santamore WP (1994) Balanc- 
ing the circulation: theoretic optimization of pulmonary/systemic flow ratio in hypoplastic left heart syndrome. J Am Coll Cardiol 24:1376-1381

3. Bove EL, Lloyd TR (1996) Staged reconstruction for hypoplastic left heart syndrome: contemporary results. Ann Thorac Surg 224: 387-395

4. Bove EL, Mosca RS (1996) Surgical repair of the hypoplastic left heart syndrome. Prog Pediatr Cardiol 5:23-35

5. Bradley SM, Mosca RS, Hennein HA, et al (1998) Bidirectional superior cavopulmonary connection in young infants. Circulation, in press

6. Donnelly J, Raffel D, Shulkin B, et al (1998) Myocardial flow reserve in human infants measured by positron emission tomography. J Thorac Cardiovasc Surg, in press

7. Fontan F, Baudet E (1971) Surgical repair of tricuspid atresia. Thorax 26:240

8. Glauser TA, Rorke LB, Weinberg PM, Clancy RR (1990) Congenital brain anomalies associated with the hypoplastic left heart syndrome. Pediatrics 85:984-990

9. Glauser TA, Rorke LB, Weinberg PM, Clancy RR (1990) Acquired neuropathologic lesions associated with the hypoplastic left heart syndrome. Pediatrics 85:991-1000

10. Goldberg CS, Schwartz EM, Brunberg JA, et al (1998) Neurodevelopmental outcome of children following the Fontan procedure. Circulation, in press

11. Gutgesell HP, Massaro TA (1995) Management of hypoplastic left heart syndrome in a consortium of university hospitals. Am J Cardiol 76:809-811

12. Helton JG, Aglira BA, Chin AJ, et al (1986) Analysis of potential anatomic or physiologic determinants of outcome of palliative surgery for hypoplastic left heart syndrome. Circulation 74(Suppl I):I-70-I-76

13. Iannettoni MD, Bove EL, Mosca RS, et al (1994) Improving results with first-stage palliation for hypoplastic left heart syndrome. J Thorac Cardiovasc Surg 107:934-940

14. Jonas RA, Lang P, Hansen D, et al (1986) First-stage palliation of hypoplastic left heart syndrome: the importance of coarctation and shunt size. J Thorac Cardiovasc Surg 92:6-13
15. Lloyd TR (1996) Prognosis of the hypoplastic left heart syndrome. Prog Pediatr Cardiol 5:57-64

16. Meliones JN, Snider AR, Bove EL, et al (1990) Longitudinal results after first-stage palliation for hypoplastic left heart syndrome. Circulation 82(Suppl IV):IV-151-IV-156

17. Mosca RS, Kulik TJ, Vermilion RP, et al (1998) Improving results with the Fontan operation for hypoplastic left heart syndrome. Circulation, in press

18. Murdison KA, Baffa JM, Farrell PE Jr, et al (1990) Hypoplastic left heart syndrome: outcome after initial reconstruction and before modified Fontan procedure. Circulation 82(Suppl IV):IV-199-IV207

19. Norwood WI, Jacobs ML, Murphy JD (1992) Fontan procedure for hypoplastic left heart syndrome. Ann Thorac Surg 54:1025-1030

20. Norwood WI, Kirklin JK, Sanders SP (1980) Hypoplastic left heart syndrome: experience with palliative surgery. Am J Cardiol 45: 87-91

21. Pigott JD, Murphy JD, Barber G, Norwood WI (1988) Palliative reconstructive surgery for hypoplastic left heart syndrome. Ann Thorac Surg 45:122-128

22. Pridjian AK, Mendelsohn A, Lupinetti FM, et al. (1993) Usefulness of the bidirectional Gleen procedure as staged reconstruction for the functional single ventricle. Am J Cardiol 71:959-962

23. Razzouk AJ, Chinnock RE, Gundry SR, Bailey LL (1996) Cardiac transplantation for infants with hypoplastic left heart syndrome. Prog Pediatr Cardiol 5:37-47

24. Reyes A, Bove EL, Mosca RS, Kulik TJ, Ludomirsky A (1997) Tricuspid valve repair in children with hypoplastic left heart syndrome during staged surgical reconstruction. Circulation 96(Suppl II)II-341-II-345

25. Rogers BT, Msall ME, Buck GM, et al (1995) Neurodevelopmental outcome of infants with hypoplastic left heart syndrome. $J$ Pediatr 126:496-498

26. Srivastava D, Preminger T, Lock JE, et al (1995) Hepatic venous blood and the development of pulmonary arteriovenous malformations in congenital heart disease. Circulation 92:1217-1222 